\title{
Auge y declive de las empresas financieras e hipotecarias de origen británico en la argentina, 1885 - 1960
}

\section{Rise and Fall of British Financial and Mortgage Enterprises in Argentina, 1885 - 1960}

\author{
Norma Silvana Lanciotti* \\ Consejo Nacional de Investigaciones Científicas y Técnicas \\ Escuela de Economía - Facultad de Ciencias Económicas y Estadística \\ Universidad Nacional de Rosario \\ (Argentina) \\ nlanciot@unr.edu.ar
}

\begin{abstract}
Resumen
Las actividades financieras e hipotecarias constituyeron uno de los principales destinos de la inversión británica en América Latina y Argentina durante la primera economía global. Estas actividades generaron elevada rentabilidad hasta la crisis de 1930, cuando la devaluación de la moneda argentina y el estancamiento de los negocios inmobiliarios marcaron el final del ciclo de inversión británico. El artículo analiza la trayectoria y la rentabilidad de las empresas británicas The River Plate E General Investment Trust, y The Mortgage Company of the River Plate, que operaron en el país desde 1888 hasta los años 1950, cuando reorientaron sus negocios a otra región. Dado que ambas firmas eran controladas por River Plate Trust, el grupo británico de inversión más importante en Argentina, se analiza la relación de estas firmas con la matriz del grupo en base a la documentación de la empresas disponible en la colección River \& Mercantile Trust Records.
\end{abstract}

LANCIOTTI, Norma Silvana "Auge y declive de las empresas financieras e hipotecarias de origen británico en la Argentina, 1885-1960”, en Avances del Cesor, Año XI, № 11, segundo semestre de 2014, pp. 175-197.

* La consulta del material documental en el cual se basa esta investigación fue posible gracias a una beca para investigadores visitantes otorgada por The British Academy, a la que agradezco.

El presente trabajo es parte del proyecto titulado "Empresas e inversiones extranjeras en la economía argentina. Un análisis histórico de largo plazo. 1870-2001”, PICT- 2010-0501/Agencia Nacional de Promoción Científica y Tecnológica, Argentina. 
Palabras clave: Inversión británica de cartera - Empresas financieras - Empresas hipotecarias - River Plate Trust - Argentina

\begin{abstract}
Financial and mortgage activities were one of the main destinations of British investment in Latin America and Argentina during the first global economy. These activities generated high returns until the crisis of 1930, when the devaluation of Argentina's currency and the stagnation of the real estate business marked the end of British investment cycle. The article analyzes the trajectory and profitability of two British companies, The River Plate \& General Investment Trust and The Mortgage Company of the River Plate, which operated in Argentina from 1888 to 1950, when they moved to other regions. Both firms were controlled by the River Plate Trust, the largest British business group during the boom of foreign investment in Argentina. The analysis will be based on files and documents of the selected companies from River \& Mercantile Trust Records.
\end{abstract}

Key Words: British Portfolio Investment - Financial companies - Mortgage companies - River Plate Trust - Argentina

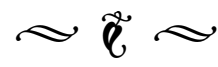

Las actividades financieras e hipotecarias constituyeron uno de los principales destinos de la inversión británica en América Latina y en Argentina durante la primera economía global. A comienzos del novecientos había comenzado el desplazamiento de la inversión británica desde Estados Unidos y los dominios británicos hacia Canadá y Argentina, los dos nuevos favoritos del mercado londinense en los albores de la Primera Guerra Mundial. La caída de la demanda de capital externo en Estados Unidos y la crisis financiera de Australia en 1893, pusieron un freno a las nuevas emisiones orientadas a dichos países, a la par que los títulos públicos y las acciones corporativas de empresas ferroviarias en Argentina aumentaban su popularidad en el mercado londinense. ${ }^{1}$ En 1913, la inversión británica en el exterior había comenzado a retroceder, salvo en Canadá y Argentina, que ocupaban respectivamente, el tercero y el cuarto lugar, entre los países receptores de inversión extranjera directa (IED). Estados Unidos y Rusia seguían encabezando la lista de países con mayor stock de IED, pero el flujo de inversiones se había desplazado hacia

1. PLATT, Desmond C. M, "The Journal of Imperial and Canada and Argentina: The first preference of the British investor, 1904-14", en The Journal of Imperial and Commonwealth History, London, 1985, V.XIII, N 3. 
Canadá y Argentina. La Primera Guerra Mundial impulsó la repatriación de capitales y la cartera de inversiones de las firmas británicas se pobló de títulos del gobierno británico, dando comienzo al declive de la entrada de capital británico en Argentina. No obstante, el país seguía ocupando el cuarto puesto entre los receptores de IED en $1938 .^{2}$

Las cifras sobre la magnitud de la inversión británica directa y de portfolio en Latinoamérica han sido muy debatidas por la historiografía británica clásica. Basándose en los directorios de empresas que cotizaban en Londres, Stone mencionaba que hacia 1913 la inversión británica en América Latina ascendía a 1.177 .462 miles de libras: $46 \%$ correspondía a inversión directa y $54 \%$ a inversiones del portfolio. ${ }^{3}$ Poco más de la mitad de las acciones correspondían a las empresas ferroviarias, el resto se repartía en compañías de servicios públicos, financieras, mineras, industriales y de transporte marítimo. Argentina encabezaba la lista de los países latinoamericanos receptores de la inversión británica, con 479.800 miles de libras: 184.593 miles de libras en títulos gubernamentales y 219.235 miles de libras en ferrocarriles (poco más de la mitad del total invertido en ferrocarriles en Latinoamérica). En Argentina la inversión directa era mayor que la inversión de portfolio, puesto que alcanzaba el 54\% del total de inversión británica en $1913 .{ }^{4}$

2. WILKINS, Mira, The History of Foreign Investment in the United States, 1914-1945, Harvard University Press, Cambridge, 2004.

3. STONE, Irving, "British Long-Term Investment in Latin America ,1865-1913”, en Business History Review, Cambridge, 1968, V. XLII: N 3 ; "British Direct and Portfolio Investment in Latin America Before 1914", en The Journal of Economic History, Cambridge, 1977, V. XXXVII, $\mathrm{N}^{\circ} 3$.

4. Íbidem.
Basándose en el trabajo pionero de Paish, ${ }^{5}$ Dunning afirmó que el $40 \%$ de la inversión de portfolio británica correspondía a acciones de las empresas ferroviarias y el 30\% a títulos de gobierno nacional y local. ${ }^{6}$ La proporción de inversión directa y de portfolio comenzó a revisarse en los años ochenta, conla redefinición de la inversión extranjera directa como aquella que involucra el control directivo de las firmas. En los cálculos de Paish, las inversiones canalizadas vía "free standing companies" habían sido consideradas como inversiones de portfolio cuando en realidad representaban inversión directa. ${ }^{7}$ Platt, también relativizó las sobreestimadas cifras de la inversión de portfolio, afirmando que hacia 1914, la mitad de las acciones de los ferrocarriles en Estados Unidos y en India ya había sido vendida a inversores locales. ${ }^{8}$ Más recientemente, Goetzmann y Ukhov, indicaron que el capital británico invertido en Argentina por la vía de títulos y acciones ascendía a 319.565 miles de libras. Aun considerando las diferencias de cálculo, la inversión de cartera representaba

5. PAISH, George, "The Export of Capital and the High Cost of Living", en The Statist (Supplement), London, 1914, No 14; "Great Britain's Capital Investments in Individual Colonial and Foreign Countries", en Journal of the Royal Statistical Society, London, 1911, V. LXXIV, No 2 .

6. DUNNING, John, Studies in International Investment, Allen \& Unwin, London, 1970.

7. CORLEY, Thomas Anthony, "Britain's Overseas Investments in 1914 Revisited”, en Business History, Cambridge, 1994, V.XXXVI, N 1; WILKINS, Mira, "The Free-Standing Company, 1870-1914: An Important Type of British Foreign Direct Investment", en Economic History Review, 1988, V.XLI, N 1.

8. PLATT, Desmond C. M., "The Journal of Imperial..." Op. Cit. 
al menos el $40 \%$ de la inversión británica en Argentina hacia $1913 .{ }^{9}$

Un segundo punto en discusión refiere a la cronología del declive de la inversión británica en el exterior y su relación con el desempeño de la economía británica. La historiografía clásica ha vinculado el cese de la inversión británica en el exterior, y particularmente en Argentina, con la desestructuración de la economía británica luego de la Primera Guerra Mundial. Después de la guerra, el ingreso de capital europeo habría cesado, a la par que se abría la puerta a la inversión norteamericana. En la visión de Platt la retirada del capital británico en la región habría sido promovida además por el creciente nacionalismo económico de los países latinoamericanos que amenazaba a las empresas británicas ${ }^{10}$. En esta línea, Jones, en su trabajo sobre el grupo Morrison / River Plate Trust, identificaba un componente político- ideológico en la retirada de las compañías, afirmando que el cambio estructural resultante de la inversión británica en Argentina paradójicamente habría provocado la reacción nacionalista de la dirigencia política local, ocasionando el fin del consenso liberal y cosmopolita previamente asumido por la oligarquía. ${ }^{11}$

9. GOETZMANN, William, UKHOV, Andrey, “British Investment Overseas 1870-1913: A Modern Portfolio Theory Approach", Working Paper 11266, NBER Working Paper Series; Cambridge, April 2005, Disponible en http://www.nber.org/papers/w11266.

10. PLATT, Desmond C. M. (editor) Business Imperialism 1840 - 1930 An Inquiry Based on British Experience in Latin America, Oxford Clarendon Press, Oxford, 1977; JONES CH, JONES Linda, GREEHILL, Robert, "Public Utility Copañies", en Íbidem.

11. JONES, Charles, "Great Capitalists and the Direction of British Overseas Investment in the Late Nineteenth Century: The Case of Argentina”, en Business History, Cambridge, 1980, V. XXII N 2.

178
Estudios más recientes han relativizado el declive de la inversión británica en Argentina durante la Primera Guerra Mundial, identificando además la persistencia del afán cosmopolita y liberal de la dirigencia política argentina que mantuvo una postura favorable al ingreso de empresas extranjeras no sólo durante la primera posguerra, sino también en los años posteriores a la crisis de $1930 .{ }^{12}$ Aun cuando en la década del veinte, la entrada de capital norteamericano se incrementaba a una tasa mayor que la tasa de ingreso del capital británico, la participación de este último en el total de la IED continuó siendo mayoritaria hasta la nacionalización de los ferrocarriles en 1947. En términos absolutos, la presencia británica en Argentina no perdió protagonismo hasta la venta de las empresas ferroviarias y de servicios públicos al estado nacional en los años cuarenta.

En trabajos anteriores sobre las empresas del grupo River Plate Trust, hemos identificado la continuidad de las inversiones después de la primera guerra mundial y la prosperidad de los negocios del grupo hasta la segunda posguerra. ${ }^{13} \mathrm{El}$ análisis de la empresa

12. LANCIOTTI, Norma, LLUCH, Andrea, "Foreign direct investment in Argentina: Timing of entry and business activities of foreign companies (1860-1950)", en Entreprises et Histoire, Paris, 2010, V. LIII; "Las empresas europeas en Argentina: condicionantes, destinos de inversión y cambios organizativos entre la Primera y la Segunda Guerra Mundial”, en Desarrollo Económico, Buenos Aires, 2012, V. LII.

13. El grupo River Plate Trust ha sido conocido como grupo Morris o Morrison, según la denominación del historiador británico Charles Jones. Estas denominaciones subrayan el protagonismo de John Morris, quien fuera presidente de RPTLA desde su creación hasta su muerte en 1905, y la posición del accionista mayoritario de la firma, Charles Morrison durante las primeras fases del 
matriz The River Plate Trust, Loan E Agency (RPTLA) y de las compañías de servicios públicos asociadas como la Compañía de Aguas corrientes de Rosario (The Rosario Waterworks Company) y la Compañía Progresos del Rosario, luego denominada Compañía de Cloacas y Desagües de Rosario (The Rosario Drainage Company), muestra la continuidad de las inversiones durante los años veinte, así como la elevada rentabilidad financiera obtenida en todos estos emprendimientos aun después de que la devaluación del peso argentino y la salida del patrón oro por la crisis de 1930, provocaran un incremento en las pérdidas por tipo de cambio. ${ }^{14}$

En esta oportunidad, nos enfocamos en las compañías del grupo River Plate Trust, dedicadas a la actividad hipotecaria y financiera. Se analiza la trayectoria y la rentabilidad de las empresas británicas The River Plate \& General Investment Trust, y The Mortgage Company of the River Plate, desde 1888 hasta los años 1950, cuando reorientaron sus negocios hacia otra región. Se identifican sus estrategias de inversión, la composición de sus carteras, la evolución del capital, reservas, activos y resultados, para determinar el grado

ciclo de vida de este grupo. Considerando que los negocios del grupo continuaron prosperando en la región del Río de la Plata durante varias décadas después de la desaparición de ambos, consideramos que el nombre River Plate Trust resulta más adecuado para definir los intereses de largo alcance del grupo en la región.

14. LANCIOTTI, Norma, "Inversión británica y redes empresariales: La estructura organizativa y las estrategias de gestión del grupo River Plate Trust, Loan E Agency en Argentina, 1881-1962", en Anuario CEEED, Buenos Aires, 2011, No 3; “ Las empresas británicas de servicios públicos en Rosario, 1885-1955”, en FRID Carina y LANCIOTTI Norma (coordinadoras) De la expansión agraria al desarrollo industrial: La economía de Santa Fe entre 1850 y 1970, Prohistoria Ediciones, Rosario, 2013. de capitalización y su rentabilidad en el largo plazo. Partiendo del supuesto de que las actividades realizadas por estas compañías, generaron elevada rentabilidad hasta la crisis de 1930, cuando la devaluación de la moneda argentina y el estancamiento de los negocios inmobiliarios marcaron el final del ciclo de inversión británico; nos proponemos indagar en las razones que determinaron que ambas compañías continuaran sus actividades en el país al menos hasta la segunda posguerra.

La investigación se basa en los archivos del fondo documental River \& Mercantile Trust Records, en el University College of London. ${ }^{15}$ Dicho fondo contiene amplia documentación sobre todas las compañías controladas por el grupo River Plate Trust. Asimismo, se consultaron los directorios de firmas y de directivos británicos en The British Library of Political E Economic Science, London School of Economics and Political Science; y los balances anuales de las compañías británicas, en The Guildhall Library, London.

Consideramos que el estudio de la trayectoria de estas empresas puede contribuir a identificar la articulación entre la inversión de portfolio y la inversión directa durante el auge de inversión británica en Argentina. Por otra parte, el estudio nos permite identificar el punto de inflexión de la tendencia de la inversión británica en Argentina y evaluar si después de la primera guerra, los capitales británicos se retiraron de Argentina como lo hicieron de Estados Unidos.

15. En 1962, The River Plate Trust, Loan E Agency se reorganiza bajo el nombre River \& Mercantile Trust Ltd., cerrando sus negocios en Argentina. 


\section{Las condiciones de la inversión en Argentina}

El atractivo de Argentina para los inversores extranjeros podía resumirse en los factores que los directivos de las firmas británicas enfatizaban repetidamente durante las asambleas de accionistas: una economía en crecimiento basada en la riqueza de sus fértiles praderas, cuya demanda creciente de crédito no podía ser cubierta con capitales locales. A medida que avanzaba el siglo veinte, se hizo evidente que el atractivo de la economía argentina se debía además a la capacidad de diversificar sus exportaciones en respuesta a la demanda europea (el rango de las productos exportados era más amplio que el de otras economías latinoamericanas), la estabilidad política y especialmente, la estabilidad financiera luego de que se estableciera nuevamente un régimen de convertibilidad del peso al oro en 1899. Estas características dieron origen a oportunidades de inversión rentable en actividades de servicios asociadas directa o indirectamente a la exportación (transporte, comunicaciones, comercio y finanzas). En consecuencia, entre 1899 y 1929, se produjo un ingreso sostenido de capitales europeos al país, bajo el paraguas de compañías financieras que cotizaban en el mercado de valores londinense.

En ese momento, la compañía financiera británica más importante en Argentina era The River Plate Trust, Loan E Agency (RPTLA). Esta firma había sido creada en 1881, para adquirir los activos y hacerse cargo del pasivo del Banco Mercantil del Río de la Plata; para prestar dinero mediante la constitución de hipotecas en la República Argentina y en el Uruguay; y asumir la gestión financiera de los negocios de compañías y particulares en el Río de la Plata. La expansión de los negocios de la firma se benefició de la composición de su staff directivo, que reunía a grandes accionistas de las empresas ferroviarias, parlamentarios, excónsules y corredores de bolsa londinenses, a inversores y comerciantes británicos residentes en Argentina y en Uruguay, además de los liquidadores y accionistas del malogrado Banco Mercantil. La presidencia estaba a cargo de John Morris, socio mayoritario de la firma Ashurst, Morris, Crisp and co., asesora legal de River Plate Trust. Morris era un abogado con amplia experiencia en la gestión financiera de compañías británicas, especialmente capacitado para diseñar esquemas de reorganización de compañías en crisis, según su experiencia adquirida en la gestión de títulos de las empresas ferroviarias británicas en Estados Unidos y Canadá durante mediados del siglo XIX. ${ }^{16}$

La expansión de la firma bajo el liderazgo de un grupo directivo con amplias conexiones en el mundo financiero londinense además de inversiones en el Río de la Plata, fundamentó la creación de nuevas empresas dedicadas a la actividad financiera e hipotecaria. Así, el éxito de plan diseñado por Morris para captar nuevos capitales en el mercado europeo, dio lugar a la constitución de dos nuevas empresas que desarrollarían las ventajas de propiedad de River Plate Trust en los negocios hipotecarios y financieros de Argentina y del Uruguay. La convocatoria captó más capital del que la firma podía incorporar en tan poco tiempo, por lo

16. Sobre Morris ver SLINN, Judy, Ashurst, Morris Crisp: A radical firm, Granta Editions, Cambridge, 1997; LANCIOTTI, Norma, "Inversión británica...", Op.Cit. Datos biográficos de Morris en Charles Jones, "Morris, John (1823-1905)", en Oxford Dictionary of National Biography, Oxford, 2004. 
cual, en 1888 se crearon River Plate E General Investment Trust y the Mortgage Company of the River Plate.

Según el acuerdo firmado entre RPTLA y The Mortgage Company of the River Plate (MCRP), la compañía se creaba para aprovechar el crecimiento de los negocios hipotecarios desarrollados por RPTLA en Argentina y en Uruguay, que habían brindado conexiones muy valiosas con prestatarios responsables, además de un excedente de capital para ser aplicado al préstamo hipotecario. Dadas las perspectivas de expansión del negocio, MCRP se constituyó en principio para prestar fondos con garantía en propiedades situadas en Argentina o en Uruguay; no obstante, según el memorándum de asociación aprobado poco después, amplió sus actividades hacia los negocios financieros. MCRP podía prestar y recibir dinero en depósito bajo cualquier término, desarrollar propiedades, comprar como principales o agentes parte o todo negocio de otras compañías, vender acciones y obligaciones de otras compañías, convirtiéndose en definitiva en una compañía hipotecaria y financiera, al igual que la empresa matriz RPTLA. ${ }^{17}$

RPTLA ejercía como agente de la nueva Mortgage, pagando sus gastos de administración y de gerenciamiento tanto en Londres como en el Río de la Plata, con excepción de los honorarios de los directores. Por su parte, Mortgage se comprometía a pagar a RPTLA una comisión del $1 \%$ de la suma prestada en hipotecas. John Morris,

17. River and Mercantile Trust Records. Agreement between The Mortgage Company of the River Plate and The River Plate Trust, Loan and Agency, June 1888; The Mortgage Company of the River Plate, Memorandum and Articles of association.
Edward Ashworth, J.H.Duncan; A.Fitz Hugh, Charles J. Gunther, William Wilson, los seis directores de Mortgage eran también directores de RPTLA. ${ }^{18}$

El ciclo de vida de MCRP se extendió por más de medio siglo. En 1946 la compañía se reorganizó bajo el nombre de The Moorside Trust ltd., cambio que terminó por formalizar la reorientación de los negocios de la compañía cuyas inversiones en tierras localizadas en el Río de la Plata, fueron desplazadas por títulos gubernamentales y acciones de compañías industriales localizadas en Gran Bretaña. ${ }^{19}$

Las condiciones que alentaron la creación de MCRP también dieron origen a The River Plate \& General Investment Trust, un fideicomiso organizado para canalizar la inversión en títulos, debentures y obligaciones gubernamentales en cualquier parte del mundo, aunque en principio, la mayoría de sus inversiones se orientaron a la Argentina y al cono sur como veremos más adelante. Dos de los cinco fideicomisarios eran directores de RPTLA. John Morris y James Anderson, presidente y secretario de RPTLA. Entre los restantes miembros, estaba Edward Thornton, ex embajador británico en Buenos Aires y Asunción, H. Doughty Browne, accionista y director del Ferrocarril del Norte de Buenos Aires, y Robert Ryrie, un hombre de negocios de la city londinense.

18. River and Mercantile Trust Records. Agreement between The Mortgage Company of the River Plate and The River Plate Trust, Loan and Agency, June 1884; The Mortgage Company of the River Plate, Memorandum and Articles of association.

19. The Moorside Trust, Memorandum of association of the Moorside trust. La composición de la cartera de inversiones en los 1950, puede verse en The Moorside Trust, Annual Report, 1961. 
En esta firma participaron los accionistas de los cuatro grandes ferrocarriles ingleses que operaban en la región. De hecho, la mayor parte de la inversión se concentraba en títulos de empresas ferroviarias en Argentina, Uruguay, Brasil, Costa Rica, México y España, luego en compañías de gas y tranvías localizadas en Estados Unidos, Argentina, Brasil, Paraguay y Chile; y finalmente en empresas de salubridad de Estados Unidos; Uruguay y Brasil. La cartera de inversiones incluía también bonos hipotecarios y bonos de gobiernos nacionales y provinciales. $^{20}$ Dado que las actividades financieras de esta firma no reconocían límites regionales desde un comienzo, River Plate E General Investment Trust no necesitó reorganizarse para seguir llevando a cabo sus negocios, sino que fue desplazando sus intereses desde Sudámerica a Gran Bretaña. La empresa actualmente sigue operando en la actividad.

En todos los casos, las empresas fueron una vía para que los inversores europeos hicieran negocios en Argentina. Esta condición era especialmente provechosa para los directivos de las compañías que además podían invertir en forma particular bajo el paraguas de la firma. Así, en 1907, se modificó el art. 58 de la sociedad MCRP, estableciendo que "los directores pueden por ellos mismos o por medio de representantes legales adquirir cualquier tipo de propiedad o constituir hipotecas". ${ }^{21}$

20. River and Mercantile Trust Records. Agreement between The River Plate and General Investment Trust Company Limited and The River Plate Trust, Loan and Agency, 14 de abril de 1888, The River Plate and General Investment Trust Company Limited, Minutes of Proceedings at fifth OGM of the shareholders, 1891, pp. 1-2.

21. Memorandum of Association of The River Plate Trust 182

\section{The Mortgage Company of the River Plate}

The Mortgage Company se creó en pleno boom inmobiliario con el objeto de ubicar el capital excedente captado en Londres. El mismo año de su creación, Murray Wilson viajó al Río de la Plata para extender los negocios hipotecarios en las provincias de Santa Fe, Buenos Aires y Entre Ríos, y en Montevideo. Al igual que en RPTLA, el criterio era no prestar a los especuladores de tierras sino a los agricultores y ganaderos. Se estipuló inicialmente que los préstamos podían alcanzar la mitad del valor de las propiedades hipotecadas, pero en razón de los disturbios en el mercado monetario de Buenos Aires, pronto se bajó el límite del préstamo a un máximo del $30 \%$ del valor de las propiedades en pesos oro. ${ }^{22}$

Los valores inmobiliarios ascendieron rápidamente hasta 1889 , cuando comenzaron su declive. La crisis de 1890 afectó los negocios de la firma sólo temporalmente. Los resultados de 1892 cayeron a la mitad de los registrados en 1890 y los cobros hipotecarios se dilataron. Hacia 1891, el valor de las propiedades había caído 40\% y seguía decreciendo. La crisis financiera puso un freno a los negocios de la compañía, que dejó de prestar por unos años. No obstante, Mortgage y RPTLA consolidaron su posición en la actividad, puesto que desplazaron a muchos de los operadores locales, que perdieron sus propiedades y abandonaron el negocio. ${ }^{23}$

Loan And Agency Company Limited. Registrado el 30 de Julio de 1881.

22. MCRP, Annual Report, 1888, 1889.

23. LANCIOTTI, Norma, "Perspectivas en crisis: Transformaciones en el comportamiento empresarial a 
Empresas Financieras e hipotecarias, 1885 - 1960...

Norma Silvana Lanciotti

Cuadro No 1. Composición del Activo de MCRP, 1888-1929 (en libras esterlinas)

\begin{tabular}{|c|c|c|c|c|c|c|}
\hline \multirow[b]{2}{*}{ AÑO } & \multicolumn{5}{|c|}{ Activo } & \multirow[b]{2}{*}{$\begin{array}{c}\text { Activo } \\
\text { Total }\end{array}$} \\
\hline & $\begin{array}{l}\text { Prestamos En } \\
\text { Hipotecas }\end{array}$ & Efectivo & $\begin{array}{l}\text { Propiedades } \\
\text { Tomadas }\end{array}$ & $\begin{array}{l}\text { Otras Inversiones } \\
\text { (Debentures) }\end{array}$ & $\begin{array}{l}\text { Prestamos } \\
\text { Temporarios } \\
\text { En Londres }\end{array}$ & \\
\hline 1888 & 200.897 & 13.877 & & & 30.000 & 252.026 \\
\hline 1890 & 804.370 & 28.775 & & 28.745 & & 870.201 \\
\hline 1892 & 665.599 & 14.343 & 106.856 & 48.143 & 20.000 & 877.927 \\
\hline 1897 & 864.425 & 16.160 & 43.300 & 64.436 & 55.000 & 1.043 .758 \\
\hline 1898 & 959.578 & 79.548 & 40.720 & 64.436 & 40.000 & 1.185 .186 \\
\hline 1900 & 1.004 .148 & 35.962 & 26.356 & 102.153 & 105.000 & 1.273 .602 \\
\hline 1902 & 1.029 .918 & 72.960 & 11.953 & 95.227 & 88.000 & 1.298 .059 \\
\hline 1904 & 1.000 .802 & 6.633 & - & 132.484 & 213.000 & 1.352 .920 \\
\hline 1906 & 1.162 .460 & 7.467 & & 157.114 & 32.000 & 1.360 .368 \\
\hline 1908 & 1.397 .717 & 70.659 & & 132.624 & 27.000 & 1.628 .001 \\
\hline 1910 & 1.466 .523 & 12.041 & & 181.185 & 58.000 & 1.717 .749 \\
\hline 1912 & 1.487 .587 & 9.374 & & 195.481 & - & 1.734 .636 \\
\hline 1914 & 1.451 .786 & 36.584 & & 205.177 & & 1.774 .966 \\
\hline 1916 & 1.273 .823 & 21.499 & 2381 & 470.105 & 4.000 & 1.816 .235 \\
\hline 1918 & 850.122 & 111.683 & & 763.812 & 4.000 & 1.779 .336 \\
\hline 1920 & 871.672 & 24.010 & & 860.634 & & 1.769 .837 \\
\hline 1922 & 801.250 & 23.420 & & 930.493 & & 1.781 .816 \\
\hline 1924 & 752.670 & 12.023 & & 1.027 .096 & & 1.807 .078 \\
\hline 1926 & 690.572 & 34.954 & & 1.102 .109 & & 1.827 .636 \\
\hline 1928 & 685.578 & 4.713 & & 1.199 .981 & & 1.904 .487 \\
\hline 1929 & 694.327 & 12.560 & & 1.160 .303 & & 1.871 .310 \\
\hline
\end{tabular}

Fuentes: MCRP, Annual Reports, 1888-1929.

partir de la experiencia de la crisis de 1890 . El caso del sector inmobiliario (Rosario, Argentina)", en História Econômica E História De Empresas, Río de Janeiro, 2006,V.
IX, No 1. Sobre la crisis de 1890 ver GERCHUNOFF, Pablo, ROCCHI, Fernado, ROSSI, Gastón, Desorden y Progreso: Las Crisis Económicas Argentinas, 1870-1905, 
En la década de 1890, se sucedieron las visitas de Farrell, Wilson y Morris a la Argentina, para evaluar la situación y la perspectiva de salida de la crisis. La evaluación de los directivos determinó la decisión de que las propiedades recibidas por deudas no se venderían en ese momento, sino que se mantendrían hasta que los precios subieran para obtener más ganancia. ${ }^{24}$ Todos se mostraban confiados sobre la evolución futura del país, y destacaban la flexibilidad y capacidad de recuperación de la economía argentina en las crisis. De hecho, en 1897, se reanudó el préstamo hipotecario y comenzaron a liquidarse las propiedades tomadas en ejecución (columna 4, cuadro № 1). Ese año los beneficios superaron a los alcanzados en 1890, marcando el comienzo de un ciclo de auge que se extendería hasta la Primera Guerra Mundial. En función de las expectativas favorables, en 1898, se decidió incrementar el capital, creando 50.000 acciones preferidas de $£ 10$ al $5 \%$. La expansión de la firma se confirma en el incremento de los préstamos hipotecarios y de otras inversiones entre 1900 y 1915, aunque claramente el núcleo de los negocios era el préstamo hipotecario, según puede observarse en el cuadro No 1 .

La participación de Gran Bretaña en la Primera Guerra Mundial, y la caída de la demanda de hipotecas en el Río de la Plata introdujeron un cambio en la cartera de inversiones de MCRP. La adquisición de títulos del gobierno británico inicialmente planteada con el fin de contribuir a financiar los gastos de guerra, se consolidó en la década

EDHASA, Buenos Aires, 2008, p. 391.

24. MCRP, Report of Proceedings at the Fifth Ordinary General Meeting of the Shareholders, January 1892. del veinte, a falta de mejores oportunidades de inversión. Entre 1918 y 1919 el negocio hipotecario estuvo prácticamente parado, pero se compraron títulos a bajo precio. La reorientación de las inversiones generó disenso entre los accionistas, quienes se preguntaban cuál era la razón para aumentar la proporción de inversiones en títulos del gobierno británico, "que rinden un 3 o $4 \%$ cuando en Argentina rinden el doble”. Los directivos del grupo afirmaban que no había posibilidades de invertir las ganancias en Gran Bretaña con más beneficio que en Argentina, pero que el superávit comercial de este país sumado al aumento de la competencia con compañías hipotecarias belgas y francesas habían hecho caer la demanda de crédito hipotecario, por lo cual la compañía optaba por priorizar la inversión segura antes que la toma de hipotecas en un mercado saturado. ${ }^{25}$

La evaluación de los directivos y accionistas era certera. Efectivamente, el rendimiento de la inversión hipotecaria en Argentina, aún en las circunstancias desfavorables de la primera posguerra era mayor al $10 \%$ sobre el capital $y$, como se puede observar en el gráfico, la firma nunca dejó de distribuir dividendos por sus acciones ordinarias. La curva de la rentabilidad financiera (ROE) muestran el período de auge del negocio entre 1898 y 1929 y posteriormente, en la década de 1950. También se observa que el rendimiento empezó a decrecer en 1933, y se mantuvo en niveles inferiores al 10\% hasta 1953 . La curva de dividendos indica que la compañía siempre pagó dividendos por sus acciones ordinarias y que éstos fueron muy elevados salvo en la década de 1930.

25. RPTLA, Report of Proceedings at the Thirty-seventh Ordinary General Meeting of the Shareholders, march 1918. 


\section{Gráfico No1. Mortgage Company of the River Plate, Rentabilidad financiera (ROE)* y dividendos distribuidos, 1888-1961}

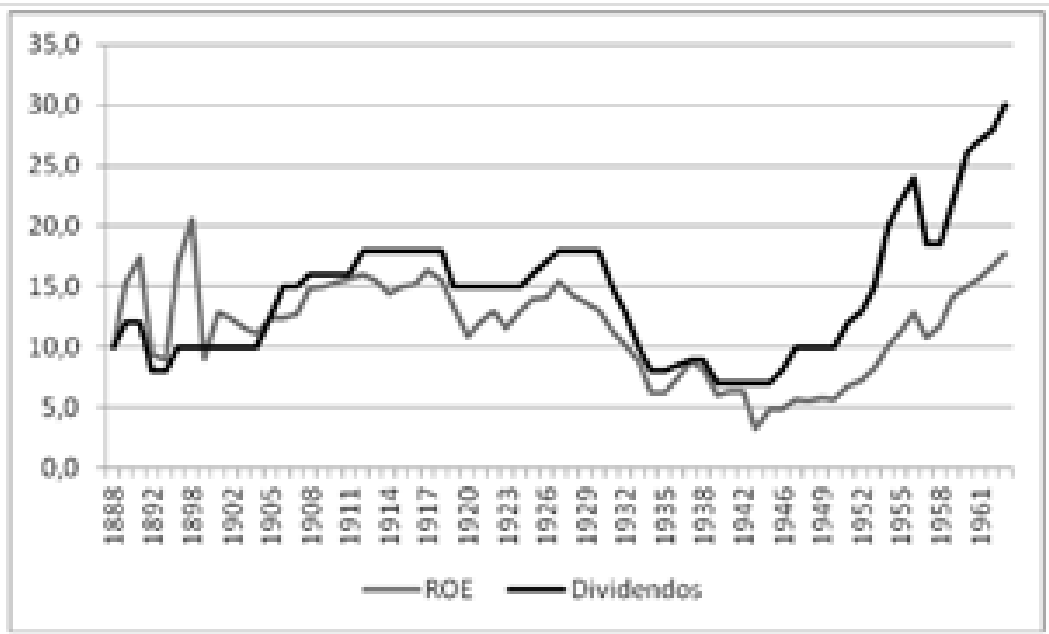

Fuente: MCRP, Annual Reports, 1888-1961

*ROE: Return on Equity. Relación entre las utilidades netas y el capital social integrado.

Durante la expansión del novecientos, la firma aumentó sus reservas hasta alcanzar el $100 \%$ del capital invertido, una política similar a la de otras compañías del grupo River Plate Trust, que sin embargo no habían alcanzado un nivel tan elevado de reservas. El nivel de reservas acumuladas tuvo su origen en las elevadas ganancias obtenidas por MCRP a partir de 1898, y permitió sostener los dividendos elevados durante todo el período. En 1919, cuando la reserva se equiparó al total del capital accionario, se decidió capitalizar parte de las ganancias acumuladas en la reserva: $£ 100.000$ fueron convertidas en 10.000 acciones ordinarias de 10 libras cada una.
Por otra parte, en lo que respecta al modo de captación de capital, se observa la preferencia por emitir obligaciones (debentures) durante el período inicial de expansión del mercado de capitales, representada en la elevada proporción de deuda sobre recursos propios (cuadro No 2, última columna). Sin embargo, los recursos propios adquirieron paulatinamente mayor protagonismo a partir de la crisis de 1930.

Al final de la Primera Guerra Mundial, las inversiones de MCRP se dirigieron a otros activos financieros en otras regiones, tal como se observa en el aumento de la participación de "otras inversiones" en el activo, en desmedro de 


\section{Cuadro No 2, Capital, resultados y rentabilidad de MCRP, 1888-1961}

\begin{tabular}{cccccccc}
\hline & $\begin{array}{c}\text { Capital social } \\
\text { Acciones } \\
\text { ordinarias }\end{array}$ & $\begin{array}{c}\text { Capital } \\
\text { acciones } \\
\text { preferidas }\end{array}$ & $\begin{array}{c}\text { Stock de } \\
\text { debentures }\end{array}$ & Reserva & $\begin{array}{c}\text { Resultado } \\
\text { del ejercicio }\end{array}$ & $\begin{array}{c}\text { ROE } \\
\text { Deuda sobre } \\
\text { recursos } \\
\text { propios* }\end{array}$ \\
\hline 1888 & 62.869 & & 178.573 & & 6.367 & 10,1 & 284,0 \\
\hline 1889 & 150.200 & & 400.000 & 50.854 & 23.040 & 15,3 & 266,3 \\
\hline 1890 & 200.000 & & 400.000 & 60.000 & 34.792 & 17,4 & 200,0 \\
\hline 1892 & 200.000 & & 400.000 & 78.000 & 18.953 & 9,5 & 200,0 \\
\hline 1894 & 200.000 & & 400.000 & 80.000 & & 0,0 & 200,0 \\
\hline 1897 & 200.000 & & 517.766 & 85.000 & 33.706 & 16,9 & 258,9 \\
\hline 1898 & 200.000 & & 521.546 & 100.000 & 41.215 & 20,6 & 260,8 \\
\hline 1900 & 200.000 & 250.000 & 521.546 & 140.000 & 41.091 & 9,1 & 115,9 \\
\hline 1902 & 200.000 & 250.000 & 521.546 & 180.000 & 55.693 & 12,4 & 115,9 \\
\hline 1904 & 200.000 & 250.000 & 514.088 & 220.000 & 50.040 & 11,1 & 114,2 \\
\hline 1906 & 200.000 & 250.000 & 511.088 & 255.000 & 55.854 & 12,4 & 113,6 \\
\hline 1908 & 200.000 & 250.000 & 688.815 & 280.000 & 66.985 & 14,9 & 153,1 \\
\hline 1910 & 200.000 & 250.000 & 693.815 & 320.000 & 68.925 & 15,3 & 154,2 \\
\hline 1912 & 200.000 & 250.000 & 693.815 & 360.000 & 71.908 & 16,0 & 154,2 \\
\hline 1914 & 200.000 & 250.000 & 693.815 & 400.000 & 64.778 & 14,4 & 154,2 \\
\hline 1916 & 200.000 & 250.000 & 693.815 & 435.000 & 68.630 & 15,3 & 154,2 \\
\hline 1918 & 200.000 & 250.000 & 693.815 & 465.000 & 70.232 & 15,6 & 154,2 \\
\hline 1920 & 300.000 & 250.000 & 656.448 & 395.000 & 59.673 & 10,8 & 119,4 \\
\hline 1922 & 300.000 & 250.000 & 655.348 & 400.000 & 71.366 & 13,0 & 119,2 \\
\hline 1924 & 300.000 & 250.000 & 655.348 & 427.644 & 71.374 & 13,0 & 119,2 \\
\hline & & & & & & $($ continúa en pág. siguiente) \\
\hline
\end{tabular}

los préstamos hipotecarios desde 1918 (cuadro No 1). Los préstamos hipotecarios continuaron disminuyendo durante los treinta hasta el final de la década, cuando representaban apenas un $18 \%$ del total del activo, cuando en el período de auge representaban el $85 \%$ del total de inversiones. Según el informe de 1940, el aumento del crédito disponible hizo caer la demanda de hipotecas, el ciclo de inversión en el mercado de hipotecas había llegado a su fin y no había expectativas favorables sobre el desarrollo futuro.

Pocos años después, el impulso del gobierno dado al Banco Hipotecario Nacional y la flexibilización de los créditos otorgados a otras entidades financieras, sumadas a la nacionalización del mercado de reaseguros, convalidaban el retiro de Mortgage del país. En 1946, la firma se reorganizó como una 


\begin{tabular}{rccccccc}
\hline & $\begin{array}{c}\text { Capital social } \\
\text { Acciones } \\
\text { ordinarias }\end{array}$ & $\begin{array}{c}\text { Capital } \\
\text { acciones } \\
\text { preferidas }\end{array}$ & $\begin{array}{c}\text { Stock de } \\
\text { debentures }\end{array}$ & Reserva & $\begin{array}{c}\text { Resultado } \\
\text { del ejercicio }\end{array}$ & $\begin{array}{c}\text { ROE } \\
\begin{array}{c}\text { Deuda sobre } \\
\text { recursos } \\
\text { propios* }\end{array}\end{array}$ \\
\hline 1926 & 300.000 & 250.000 & 655.348 & 455.000 & 77.450 & 14,1 & 119,2 \\
\hline 1928 & 300.000 & 300.000 & 650.348 & 425.000 & 85.607 & 14,3 & 108,4 \\
\hline 1930 & 300.000 & 300.000 & 650.348 & 455.000 & 78.196 & 13,0 & 108,4 \\
\hline 1932 & 300.000 & 300.000 & 650.348 & 470.000 & 61.155 & 10,2 & 108,4 \\
\hline 1934 & 300.000 & 300.000 & 650.348 & 475.000 & 37.095 & 6,2 & 108,4 \\
\hline 1936 & 300.000 & 300.000 & 650.348 & 475.000 & 44.066 & 7,3 & 108,4 \\
\hline 1938 & 300.000 & 300.000 & 650.348 & 305.000 & 48.838 & 8,1 & 108,4 \\
\hline 1940 & 300.000 & 300.000 & 645.448 & 305.000 & 38.376 & 6,4 & 107,6 \\
\hline 1942 & 300.000 & 300.000 & 645.248 & 305.000 & 37.837 & 6,3 & 107,5 \\
\hline 1944 & 300.000 & 300.000 & 645.248 & 305.000 & 19.637 & 3,3 & 107,5 \\
\hline 1946 & 300.000 & 300.000 & 645.248 & 322.789 & 29.472 & 4,9 & 107,5 \\
\hline 1948 & 300.000 & 300.000 & 645.248 & 338.591 & 33.393 & 5,6 & 107,5 \\
\hline 1950 & 300.000 & 300.000 & 645.000 & 397.083 & 34.129 & 5,7 & 107,5 \\
\hline 1952 & 300.000 & 300.000 & 645.000 & 423.049 & 43.223 & 7,2 & 107,5 \\
\hline 1954 & 300.000 & 300.000 & 645.000 & s.d & 60.521 & 10,1 & 107,5 \\
\hline 1956 & 363.000 & 300.000 & 700.000 & s.d & 85.042 & 12,8 & 105,6 \\
\hline 1958 & 600.000 & 300.000 & 700.000 & 571.000 & 106.147 & 11,8 & 77,8 \\
\hline 1960 & 735.000 & 300.000 & 700.000 & 645.000 & 154.871 & 15,0 & 67,6 \\
\hline 1962 & 735.000 & 300.000 & 700.000 & s.d & 172.393 & 16,7 & 67,6 \\
\hline 1963 & 808.500 & 300.000 & 700.000 & 859.000 & 196.604 & 17,7 & 63,1 \\
\hline & & & & & & & \\
\hline
\end{tabular}

Fuente: MCRP, Annual Reports, 188-1963

*. Deuda/Recursos Propios. Indica el grado de endeudamiento.

Es la relación entre el capital obligatorio (debentures) y el capital accionario.

financiera bajo la denominación The Moorside Trust Limited. La reforma de los estatutos redefinió a su actividad principal: invertir fondos con garantía en propiedades al igual que su antecesora, pero sin limitar el área de operaciones a Argentina y Uruguay. En la reunión de accionistas de 1947, el presidente de la compañía, Walter Woodbine Parish, planteaba que el préstamo con hipotecas ya no era rentable en Argentina ni en Gran Bretaña, puesto que la demanda de crédito era cubierta por otros mecanismos y las tasas de interés ya no eran tan remunerativas como antes, a partir de lo cual se disponía la reducción del capital. ${ }^{26}$ En 1949, comenzó la liquidación de los activos de Moorside trust en Argentina. ${ }^{27}$ Como observamos en el cuadro siguiente, la

26. MCRP, Annual General Meeting, 1947.

27. Moorside Trust fue adquirido por Guinness Peat en 1984. 


\section{Cuadro No 3. Distribución de las inversiones \\ de Moorside Trust, 1948-1949, 1954-1955}

\begin{tabular}{lcccc}
\hline $\begin{array}{l}\text { Clasificación de las Inversiones } \\
\text { según Costo de Libros }\end{array}$ & $\mathbf{1 9 4 8}$ & $\mathbf{1 9 4 9}$ & $\mathbf{1 9 5 4}$ & $\mathbf{1 9 5 5}$ \\
\hline $\begin{array}{l}\text { Debenture and British } \\
\text { Government Stocks }\end{array}$ & 12,44 & 6,95 & 1,5 & 0,99 \\
Preference stocks and shares & 34,06 & 34,65 & 18,68 & 13,6 \\
Ordinary stocks and shares & 53,5 & 58,4 & 79,82 & 85,41 \\
\hline
\end{tabular}

Distribución Geográfica de las inversiones

\begin{tabular}{lcccc}
\hline UK & 77,08 & 79,03 & 79,78 & 79,72 \\
Dominios y Colonias & 10,69 & 11,12 & 8,64 & 7,36 \\
Sudamérica & 7,67 & 6,11 & 2,3 & 1,32 \\
Otros países extranjeros & 3,76 & 3,74 & 1,66 & 1,72 \\
USA y Canadá & & & 7,62 & 9,88
\end{tabular}

Fuente: MCRP, Annual Reports, 1948, 1949, 1954, 1955

estructura de las inversiones había variado radicalmente, tanto en su composición como en su distribución geográfica y hacia 1955, la compañía ya no tenía intereses en Argentina.

\section{The River Plate E General Investment Trust}

The River Plate \& General Investment Trust (RP \& GIT) también se creó en 1888. Según el acuerdo entre la firma y RPTLA, ésta se haría cargo del lanzamiento del fideicomiso (incluyendo los gastos preliminares de preparación del prospectus, su publicación y circulación), y de su administración, mientras que RP \& GIT pagaría una comisión del 1\% sobre el capital suscripto. ${ }^{28}$ En su carácter de agente, RPTLA pagaría todos los gastos de administración del trust, incluyendo la remuneración de los trustees (socios fiduciarios), mientras que el trust se comprometía a transferir una comisión anual del 5\% sobre el capital aportado.

La organización de un fideicomiso para administrar las propiedades de los fiduciantes manifestaba relaciones de alta confianza entre las partes dado que este tipo de organización implica la transferencia de la titularidad de bienes. En efecto, los fiduciantes eran directores y accionistas de RPTLA y los trustees de RP \& GIP, H. Doughty Browne,

28. Agreement between The River Plate and General Investment Trust Company Ltd. and the River Plate Trust, Loan E Agency Co. Ltd., 14 de abril de 1888. 
A.J. Fitzghugh, John Morris, Robert Ryrie, y Edward Thornton, eran también directivos de RPTLA. En las décadas siguientes, los trustees no siempre fueron directivos de la matriz, pero sí lo eran de otras empresas del grupo. El carácter subsidiario de River Plate E General Investment Trust fue mencionado por el Secretario James Anderson en la primera asamblea de accionistas, aludiendo a que la conexión con RPTLA permite a la compañía adquirir en términos favorables inversiones seguras, "by continuing the policy of investing only in well-selected securities". ${ }^{29}$

¿Cuáles eran los valores seleccionados? Además de acciones de otras empresas de grupo, RP \& GIT adquirió en 1888, debentures y acciones de compañías ferroviarias en América Latina, España y Estados Unidos, de compañías de gas, salubridad y telefónicas en América Latina, títulos y obligaciones del gobierno de Costa Rica, del gobierno de la provincia de Entre Ríos y del gobierno de la ciudad de Buenos Aires. Más allá de la aparente diversificación de la cartera de inversiones, el $86 \%$ de éstas correspondía a empresas que operaban en el Río de la Plata. Más notablemente aún, casi el 60\% del monto invertido en Argentina y Uruguay correspondía a préstamos otorgados a comerciantes y terratenientes de la región pampeana argentina (Ramayon, Pellicer, Mason, Constantino Raffo, Francisco Laforcada y Thomas Armstrong). De modo que al comienzo, la política del trust no siguió los criterios generales establecidos por RPTLA en cuanto a evitar los préstamos a terratenientes y especuladores, y diversificar la cartera.

29. The River Plate E General Investment Trust, Annual Report, Second Ordinary General Meeting, January 1889.

\section{Cuadro No 4: Cartera de inversiones de River Plate \& Investment Trust, 1888}

\begin{tabular}{lc}
\hline \multicolumn{2}{c}{ Compra de acciones y debentures } \\
\hline Argentine Northen Central Extension & 4.706 \\
Costa Rica Gov. & 5.684 \\
\hline Cordoba Central Railway & 5.252 \\
\hline Taltal Railway & 4.367 \\
\hline East Argentine Railway Stock & 5.818 \\
\hline Leopoldina & 8.966 \\
\hline Cucuta Railway & 5.000 \\
\hline Alabama Great Southern Railroad & 1.900 \\
\hline Buenos Aires E Pacific Railway & 6.549 \\
\hline Mortgages In The River Plate & 20.000 \\
\hline River Plate Trust Loan And Agency. A Shares & 3.540 \\
\hline River Plate Trust Loan And Agency B Shares & 3.535 \\
\hline Taltal Railway & 200 \\
Buenos Aires E Pacific Railway & 5.000 \\
\hline Taltal Railway & 200 \\
\hline Madero Port Works Trust & 5.000 \\
\hline Central Argentine Railway Ordinary Stock & 5.640 \\
\hline Belgrano And Buenos Aires Gas Shares & 1.225 \\
\hline Buenos AiresGreat Southern Railway Ordinary & 3.952 \\
Stock & \\
\hline Buenos Aires New Gas Company & 100 \\
\hline Mortage Co. of The River Plate & 5.965 \\
\hline Buenos Aires E Rosario Railway & 4.244 \\
\hline United River Plate Telephone & 1.000 \\
\hline Central Argentine Railway Ordinary Stock \\
Belgrano And Buenos Aires Gas Shares & 2.815 \\
\hline Buenos Aires Great Southern & 100 \\
Buenos Aires E Rosario Railway & 2.000 \\
\hline West African Telegraph & 2.500 \\
\hline Algeciras (Gibraltar) Railway & 5.000 \\
\hline Uruguay Government & 500 \\
\hline Great Westerm Railway Of Brazil & 5.000 \\
\hline Buenos Aires Central Produce Market & 5.000 \\
\hline Central Produce Market Of BA & 2.600 \\
\hline Foreign External Debt Of Entre Rios & \\
\hline
\end{tabular}

(continúa en página siguiente) 
Interoceanic Railway Of Mexico

2.703

City F BA Loan

3.178

Rosario WaterWorks

10.000

Great Southern Of Spain

5.000

Northern Railway Of BA

3.630

Rosario Improvements

5.000

Entre Rios Central Railway Extension 4.000

(Mortgage Bonds)

Cantaneira Water Supply Dranaige. Sao Paulo.

5.000

Central Uruguay Northern Extension 5.000

Railway Co.

Montevideo Waterworks Co.

10.000

Préstamos

\begin{tabular}{lc}
\hline Ramayon & 40.000 \\
Pellicer & 20.000 \\
Mason & 78.000 \\
\hline Constantino Raffo & 5.000 \\
\hline Francisco Laforcada & 20.000 \\
Armstrong & 40.000 \\
TOTAL & 399.698 \\
\hline
\end{tabular}

Fuente: River Plate E General Investment Trust, Minutes Book, 1888. con lo aconsejado por Morris, las inversiones crecieron ininterrumpidamente en la década del noventa, en la medida en que la compañía aprovechaba para adquirir títulos a valores depreciados (cuadro No 5). Edward Thornton, uno de los socios fiduciarios del trust, tranquilizaba a los accionistas afirmando que sus inversiones eran seguras y remunerativas, porque no habían invertido en aquellas actividades que pudieran ser afectadas por las decisiones de los gobiernos locales después de la crisis, como en las empresas de tramways, por ejemplo. ${ }^{31}$

La promoción del trust como negocio seguro se representaba en la composición de su capital, integrado por acciones diferidas y preferidas en partes iguales. La característica de las acciones diferidas es que devengan un buen rendimiento a la par que mantienen un precio estable en el mercado. Esto implica ganancias de capital no tan elevadas pero sin riesgos derivados de la caída brusca del valor de los títulos y acciones ordinarias. Las acciones preferidas, también ofrecen una mayor seguridad de cobro a los tenedores, en tanto tienen preferencia en el cobro de dividendos.

No todos los accionistas parecían acordar

En 1889, siguiendo las recomendaciones que John Morris enviaba por escrito, comenzaron a vender a precios elevados muchos de los valores adquiridos el año anterior, especialmente acciones de empresas ferroviarias y los títulos del empréstito de la ciudad de Buenos Aires. La crisis de 1890 no impactó negativamente en la firma, sino que por el contrario atrajo nuevas oportunidades de negocios. Como decían Morris y Van Raalte, era el momento de comprar títulos, puesto que estaban a bajo precio. ${ }^{30}$ En línea

30. River Plate E General Investment Trust; Minutes of con las decisiones tomadas por los trustees, especialmente con aquellas tomadas durante el boom especulativo de los 1880. En la asamblea

Proceedings at the Fifth Ordinary General Meeting of the Shareholders, February 1892.

31. River Plate \& General Investment Trust; Minutes of Proceedings at the Fifth Ordinary General Meeting of the Shareholders, February 1892. La aseveración de Thornton no era cierta, de todos modos, ya que el trust había invertido en la empresa de cloacas de Rosario (The Rosario Drainage Co.), que enfrentó un extendido conflicto con el gobierno local por incumplimiento de las inversiones pautadas en contrato. 
de accionistas de 1893, hubo discusiones por la desvalorización de los títulos comprados a precio de boom. P.W. Carr reclamaba las excesivas sumas invertidas por los directores en pocas opciones, y sobre todo, la preferencia por las acciones en ferrocarriles, que no le parecían una buena opción. A esta queja, se sumaba la interpelación de otro accionista que quería informarse acerca de cuánto efectivamente se habían depreciado los valores en los que había invertido la compañía. Las quejas cesaron cuando Carr fue designado socio fiduciario en 1893. ${ }^{32}$

A partir de 1896, siempre asesorados por Morris, el Trust comenzó a tomar acciones y debentures de varios holdings eléctricos (River Plate Electric, Light and Traction; River Plate Electricity Co., City of London Electric Lightning, British Electric Traction, Urban Electric Supply, Rosario Electric Co., Córdoba Light and Power). ${ }^{33}$ Las compras se incrementaron notablemente a partir del novecientos, y en 1907 se emitieron debentures para captar capital adicional. También las ganancias netas se incrementaron rápidamente hasta llegar al máximo en 1913.

El estallido de la Primera Guerra Mundial limitó temporariamente los negocios de la compañía y una vez finalizada la guerra, la tendencia ascendente se acentuó. En 1918, de modo similar a lo actuado por Mortgage, los trustees recomendaron la capitalización de parte de la ganancia acumulada en la reserva: $£$ 50.000 libras en acciones diferidas de $£ 2$ cada una. A partir de entonces, se retomó el ciclo de auge de los negocios de River Plate \& General

32. River Plate E General Investment Trust; Minutes of Proceedings at the Sixth Ordinary General Meeting of the Shareholders, February 1893.

33. River Plate E General Investment Trust, Minutes Book.
Investment Trust que se extendió hasta 1930, como podemos observar en el cuadro No 5 .

Los dividendos a las acciones diferidas distribuidos por RP\&GIT, siempre fueron más elevados que aquellos repartidos por otros trusts o compañías holding similares, con excepción de los dividendos distribuidos por la matriz del grupo, RPTLA que llegaban al $24 \%$ en la década de 1920 . Por ejemplo, en principio River Plate Electricity, distribuyó dividendos equivalentes a los repartidos por RP\&GIT, pero a las acciones ordinarias de las cuales se espera un premio mayor. Después de 1910, sus dividendos siempre fueron dos puntos menos que los devengados por el trust. Su sucesora River Plate Electricity and Other Securities Corporation, creada en 1937 siempre repartió un porcentaje menor de dividendos que RP\& GIT. The Nerw Zealand E River Plate Land E Mortgage Co, creada en 1883 (8\% en 1926) y The London trust (1894: 4\%. 19041906: 2\% 1908-9: 3, 1918-19: 8; 1920-22: 10, 1922-23:12, 1924: 12; 1928: 18) repartían aún menos dividendos. Omnium Investments, tenía el doble de capital y rendía la mitad que el RP \& GIT en los $1920 .{ }^{34}$

Luego de la caída de la actividad como consecuencia de la crisis de 1930 primero y por la Segunda Guerra Mundial, después, se inició un segundo ciclo de auge, que en los 1950s alcanzaría rentabilidades aún superiores a las obtenidas en los años 1920 (ROE, cuadro No 5). Ahora bien, para entonces la cartera de inversiones se había desplazado desde Sudamérica hacia Gran Bretaña. En 1938,31 \% de las inversiones permanecían en Sudamérica,

34. Annual Reports de varias compañías. The Guildhall Lybrary. Corporate Reports, London. 
Cuadro No 5: Capital, activo, reservas y utilidades de The River Plate \& General Investment Trust, 1888-1961

\begin{tabular}{|c|c|c|c|c|c|c|c|c|}
\hline \multirow[t]{2}{*}{ Año } & \multicolumn{2}{|c|}{ Capital social } & \multirow{2}{*}{$\begin{array}{l}4 \% \\
\text { Debenture } \\
\text { stock }\end{array}$} & \multirow[b]{2}{*}{ Reserva } & \multirow{2}{*}{$\frac{\text { Activo }}{\text { En inversiones }}$} & \multirow{2}{*}{$\begin{array}{l}\text { Utilidades } \\
\text { Netas }\end{array}$} & \multirow[b]{2}{*}{ ROE } & \multirow{2}{*}{$\begin{array}{c}\text { Dividendos } \\
\text { acciones } \\
\text { diferidas }\end{array}$} \\
\hline & $\begin{array}{c}\text { Preferred } \\
\text { stock }\end{array}$ & $\begin{array}{c}\text { Deferred } \\
\text { Stock }\end{array}$ & & & & & & \\
\hline 1888 & & & & & 208.059 & & & 5,0 \\
\hline 1889 & 250.000 & 250.000 & & 5.246 & 443.442 & 18.158 & 4 & 5,0 \\
\hline 1890 & 250.000 & 250.000 & & 11.633 & 514.681 & 25.388 & 5,08 & 4,5 \\
\hline 1891 & 250.000 & 250.000 & & 16.513 & 510.400 & 17.494 & 3,50 & 2,0 \\
\hline 1892 & 250.000 & 250.000 & & 18.282 & 523.522 & 16.526 & 3,31 & 2,0 \\
\hline 1894 & 250.000 & 250.000 & & 12.830 & 524.364 & 16.925 & 3,39 & 2,0 \\
\hline 1895 & 250.000 & 250.000 & & 14.381 & 542.777 & 19.131 & 3,83 & 2,5 \\
\hline 1897 & 250.000 & 250.000 & & 23.000 & 550.285 & 18.989 & 3,80 & 3,0 \\
\hline 1898 & 250.000 & 250.000 & & 20.920 & 536.865 & 20.188 & 4,04 & 3,0 \\
\hline 1899 & 250.000 & 250.000 & & 28.044 & 544.683 & 21.302 & 4,26 & 3,5 \\
\hline 1900 & 250.000 & 250.000 & & 28.983 & 536.446 & 23.066 & 4,61 & 3,5 \\
\hline 1901 & 250.000 & 250.000 & & 26.000 & 538.712 & 22.067 & 4,41 & 3,5 \\
\hline 1902 & 250.000 & 250.000 & & 14.000 & 552.781 & 23.457 & 4,69 & 3,5 \\
\hline 1904 & 250.000 & 250.000 & & 32.500 & 541.365 & 25.240 & 5,05 & 4,5 \\
\hline 1906 & 250.000 & 250.000 & & 60.000 & 575.447 & 29.853 & 5,97 & 6,0 \\
\hline 1907 & 250.000 & 250.000 & 150.000 & 62.998 & 729.948 & 31.000 & 6,20 & 6,5 \\
\hline 1910 & 250.000 & 250.000 & 250.000 & 106.000 & 910.389 & 39.832 & 7,97 & 9,0 \\
\hline 1912 & 250.000 & 250.000 & 250.000 & 145.000 & 961.701 & 45.398 & 9,08 & 11,0 \\
\hline 1913 & 250.000 & 250.000 & 250.000 & 150.000 & 955.704 & 48.413 & 9,68 & 11,0 \\
\hline 1914 & 250.000 & 250.000 & 250.000 & 150.000 & 966.449 & 42.592 & 8,52 & 11,0 \\
\hline 1915 & 250.000 & 250.000 & 250.000 & 160.000 & 961.456 & 40.505 & 8,10 & 10,0 \\
\hline 1916 & 250.000 & 250.000 & 250.000 & 164.000 & 949.956 & 40.858 & 8,17 & 10,0 \\
\hline 1918 & 250.000 & 250.000 & 250.000 & 172.000 & 965.303 & 44.353 & 8,87 & 10,0 \\
\hline 1919 & 275.000 & 275.000 & 250.000 & 126.000 & 987.700 & 46.692 & 8,49 & 10,0 \\
\hline 1920 & 275.000 & 275.000 & 250.000 & 130.000 & 986.095 & 53.609 & 9,75 & 10,0 \\
\hline 1921 & 275.000 & 275.000 & 250.000 & 33.390 & 1.042 .079 & 47.699 & 8,67 & 10,0 \\
\hline 1922 & 275.000 & 275.000 & 250.000 & 38.390 & 1.065 .226 & 51.254 & 9,32 & 11,0 \\
\hline 1923 & 275.000 & 275.000 & 250.000 & 43.390 & 1.052 .624 & 56.598 & 10,29 & 12,0 \\
\hline 1924 & 275.000 & 275.000 & 250.000 & 48.390 & 1.069 .928 & 60.657 & 11,03 & 14,0 \\
\hline 1925 & 275.000 & 275.000 & 250.000 & 75.000 & 1.098 .951 & 64.717 & 11,77 & 16,0 \\
\hline
\end{tabular}

(continúa en página siguiente) 


\begin{tabular}{|c|c|c|c|c|c|c|c|c|}
\hline \multirow[b]{2}{*}{ Año } & \multicolumn{2}{|c|}{ Capital social } & \multirow{2}{*}{$\begin{array}{l}4 \% \\
\text { Debenture } \\
\text { stock }\end{array}$} & \multirow[b]{2}{*}{ Reserva } & \multirow{2}{*}{$\frac{\text { Activo }}{\text { En inversiones }}$} & \multirow{2}{*}{$\begin{array}{l}\text { Utilidades } \\
\text { Netas }\end{array}$} & \multirow[b]{2}{*}{ ROE } & \multirow{2}{*}{$\begin{array}{c}\text { Dividendos } \\
\text { acciones } \\
\text { diferidas }\end{array}$} \\
\hline & $\begin{array}{c}\text { Preferred } \\
\text { stock }\end{array}$ & $\begin{array}{c}\text { Deferred } \\
\text { Stock }\end{array}$ & & & & & & \\
\hline 1926 & 275.000 & 275.000 & 250.000 & 80.000 & 1.149 .707 & 67.899 & 12,35 & 17,5 \\
\hline 1927 & 275.000 & 275.000 & 250.000 & 85.000 & 1.207.238 & 72.090 & 13,11 & 20,0 \\
\hline 1928 & 275.000 & 275.000 & 250.000 & 89.000 & 1.256 .767 & 74.966 & 13,63 & 20,0 \\
\hline 1929 & 275.000 & 275.000 & 250.000 & 95.000 & 1.317 .244 & 78.329 & 14,24 & 20,0 \\
\hline 1930 & 275.000 & 275.000 & 250.000 & 100.000 & 1.363 .588 & 76.616 & 13,93 & 20,0 \\
\hline 1931 & 275.000 & 275.000 & 250.000 & 105.000 & 1.312 .345 & 62.231 & 11,31 & 16,0 \\
\hline 1932 & 275.000 & 275.000 & 250.000 & 110.000 & 1.312 .526 & 48.231 & 8,77 & 12,0 \\
\hline 1933 & 275.000 & 275.000 & 250.000 & 112.500 & 1.285 .757 & 40.993 & 7,45 & 10,0 \\
\hline 1934 & 275.000 & 275.000 & 250.000 & 112.500 & 1.344 .882 & 42.326 & 7,70 & 10,0 \\
\hline 1935 & 275.000 & 275.000 & 250.000 & 115.000 & 1.346 .903 & 42.642 & 7,75 & 10,0 \\
\hline 1936 & 275.000 & 275.000 & 250.000 & 115.000 & 1.368 .445 & 43.909 & 7,98 & 10,0 \\
\hline 1937 & 275.000 & 275.000 & 250.000 & 117.500 & 1.393 .008 & 52.354 & 9,52 & 10,0 \\
\hline 1938 & 275.000 & 275.000 & 250.000 & 122.500 & 1.447 .084 & 47.971 & 8,72 & 10,0 \\
\hline 1939 & 275.000 & 275.000 & 250.000 & 130.000 & 1.381 .415 & 45.065 & 8,19 & 10,0 \\
\hline 1940 & 275.000 & 275.000 & 250.000 & 133.000 & 1.371 .433 & 44.088 & 8,02 & 10,0 \\
\hline 1941 & 275.000 & 275.000 & 250.000 & 135.000 & 1.361 .561 & 43.069 & 7,83 & 10,0 \\
\hline 1942 & 275.000 & 275.000 & 250.000 & 139.000 & 1.344 .984 & 43.429 & 7,90 & 10,0 \\
\hline 1944 & 275.000 & 275.000 & 250.000 & 143.000 & & 58.902 & 10,71 & 10,0 \\
\hline 1945 & 275.000 & 275.000 & 250.000 & 153.000 & 1.218 .608 & 60.680 & 11,03 & 11,0 \\
\hline 1947 & 275.000 & 275.000 & 250.000 & 165.000 & 1.221 .001 & 67.671 & 12,30 & 12,0 \\
\hline 1949 & 275.000 & 275.000 & 250.000 & 185.000 & & 71.375 & 12,98 & 14,0 \\
\hline 1950 & 275.000 & 275.000 & 250.000 & 192.500 & 1.260 .782 & 74.223 & 13,50 & 14,0 \\
\hline 1952 & 275.000 & 275.000 & 250.000 & 203.000 & & 89.919 & 16,35 & 17,0 \\
\hline 1953 & 275.000 & 275.000 & 250.000 & & & 95.336 & 17,33 & 19,0 \\
\hline 1955 & 275.000 & 550.000 & 250.000 & 200.000 & 1.318 .905 & 128.450 & 15,57 & 14,0 \\
\hline 1957 & 275.000 & 605.000 & 250.000 & 235.000 & & 155.617 & 17,68 & 17,0 \\
\hline 1958 & 275.000 & 605.000 & 450.000 & 254.000 & 1.599 .180 & 171.429 & 19,48 & 18,5 \\
\hline 1961 & 275.000 & 605.000 & 450.000 & 375.564 & 1.721 .639 & 264.163 & 30,02 & 28,0 \\
\hline
\end{tabular}

Fuentes: The River Plate \& General Investment Trust, Annual Reports, 1888-1961; Minutes Books, 1888-1961. 
mientras $41 \%$ estaba en Gran Bretaña. ${ }^{35}$ Un informe de 1945 mencionaba que en Argentina las oportunidades de inversión se mantenían difíciles, puesto que el rendimiento del capital y los intereses habían caído, y que si bien se apreciaba el incremento de los dividendos, "we have had repayments and conversions to lower rates of interest in the case of first class debenture stocks, which had previously shown us a a good return of the money invested. ${ }^{136} \mathrm{La}$ imagen pesimista se completaba con un comentario poco auspicioso que preveía la reducción de los ingresos por la nacionalización, los efectos de la escasez de carbón y "ferver opportunities of remunerative employment of surplus funds cannot but have serious repercussions on investment trust companies such as ours." 37

El acuerdo para la transferencia de las compañías ferroviarias, de gas y salubridad al estado argentino, mermó aún más los intereses del trust en Argentina. Hacia 1947, la participación sudamericana en el total de inversiones se había reducido al $17 \% .^{38}$ Pocos meses más tarde el presidente de la compañía, Charles Pikington aseveraba que el portfolio de inversiones sería afectado por los programas de nacionalización pero no de manera radical ya que las tenencias de títulos estaban repartidas en diferentes sectores y en grupos pequeños, por lo que la pérdida no sería significativa. También se mencionaba, que luego del acuerdo entre Gran Bretaña y Argentina, se

35. River Plate \& General Investment Trust Co, Annual Report, 1950.

36. River Plate \& General Investment Trust Co. Minute Ordinary General Meeting, 24th February 1947.

37. Íbidem.

38. El $64 \%$ de las tenencias del trust estaban en Gran Bretaña y $12 \%$ en países del Commonwealth. ${ }^{-}$River Plate E General Investment Trust co, Annual Report, 1950. esperaba recibir el efectivo proveniente de los holdings en los ferrocarriles, aproximadamente 18.000 libras. ${ }^{39}$ La venta de los ferrocarriles y de las compañías de salubridad de Rosario al Estado Argentino así como la venta de Montevideo WaterWorks Company al estado uruguayo redujeron los holdings de RP\&GIT en Sudamérica, marcando el declive absoluto de la presencia británica en el Río de la Plata. Hacia 1958, el trust ya no tenía ninguna inversión en Sudamérica. Cuando RPTLA se reorganizó como The River \& Mercantile Trust $L t d$. ambas empresas terminaron su relación para operar como trusts independientes.

\section{Compañías británicas en Argentina: Comentarios finales}

La cronología de la relación entre Gran Bretaña y Argentina no puede prescindir del análisis de la inversión de portfolio que ingresó al Río de la Plata por la vía de las empresas financieras e hipotecarias que cotizaban en el mercado de valores londinenses. El análisis de la trayectoria de las firmas nos permite revisar la cronología de las relaciones económicas anglo-argentinas y la idea del declive británico de posguerra. Las transformaciones económicas resultantes de la desestructuración de la primera economía global ocasionada por 1a Primera Guerra Mundial, generaron las condiciones para el repliegue de la inversión británica en Sudamérica. No obstante, el estudio de estos casos nos permite confirmar que la entrada de capital europeo por vía de la inversión de cartera cesó efectivamente a la salida de la guerra, en el momento en que se consolidó el movimiento inverso de capitales

39. Notes for chairman's speech at the 61st ordinary general meeting to be held on February 23, 1948. 
desde Sudamérica hacia el norte europeo por vía de las utilidades repartidas.

Vemos que en ambas compañías el flujo de capitales por dividendos hacia Gran Bretaña se intensificó en la primera posguerra hasta 1930, sumando un monto que triplicaba el monto de los capitales inicialmente invertidos. Asimismo, en los años 1930, la transferencia de utilidades de River Plate E General Investment Trust continuó siendo elevada, nunca por debajo del $10 \%$ a las acciones diferidas. Considerando la cartera de inversiones de ambas compañías en la década del treinta, se establece que dichos rendimientos tenían origen en los valores invertidos en la Argentina. En la Segunda Guerra Mundial, se produjo el segundo momento de inflexión hacia el desplazamiento definitivo de la inversión hacia el norte europeo. La nueva fase de aumento de las inversiones y de los rendimientos que comenzó en la segunda posguerra ya no dependía del rendimiento de los recursos sudamericanos, dado que los intereses se habían reducido al mínimo.

En cuanto a la capitalización, ambas compañías aplicaron la política del grupo River Plate Trust ya identificada en otras compañías. Priorizaron la captación del

Gráfico No 2: Mortgage Co. of the River Plate y River Plate E General Investment: Rentabilidad financiera, 1888-1958.

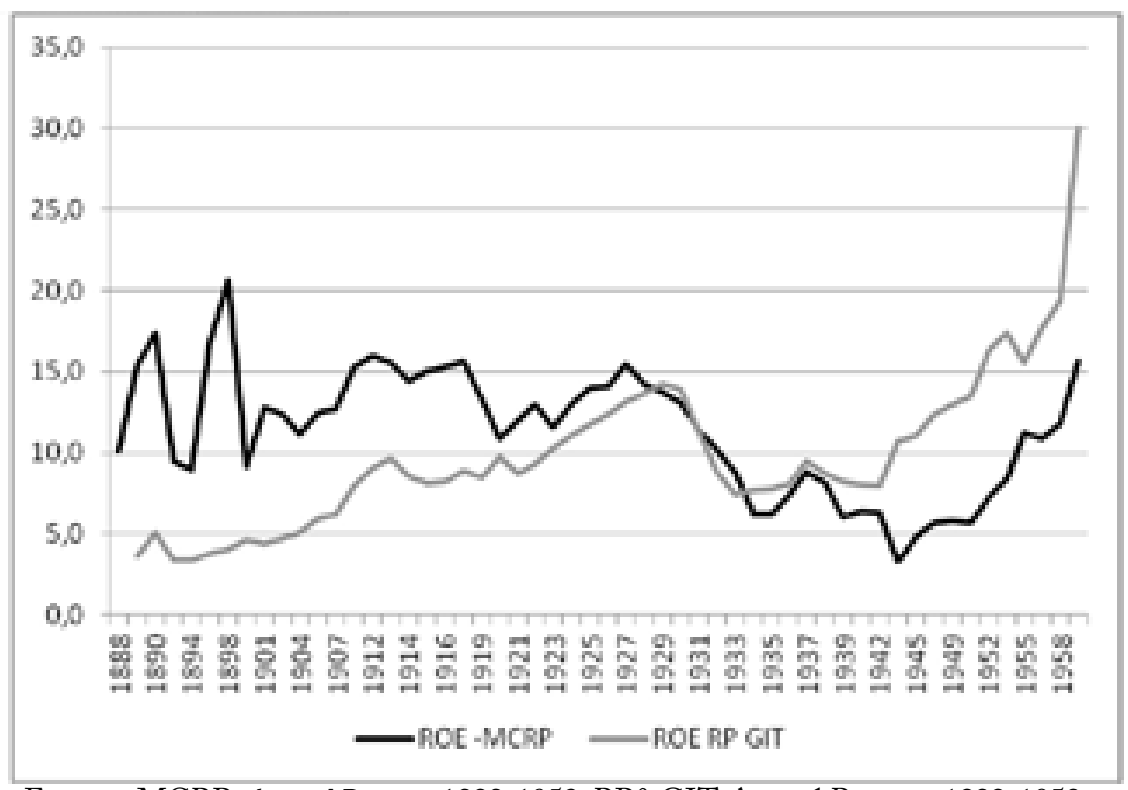

Fuentes: MCRP, Annual Reports, 1888-1958; RP\&GIT, Annual Reports, 1888-1958 
capital vía emisión de debentures cuando los rendimientos eran bastante más elevados que los intereses a pagar. Cuando los intereses aumentaron, se financiaron con las ganancias acumuladas en las reservas. Ambas empresas estaban fuertemente capitalizadas y nunca interrumpieron el pago de dividendos.

$\mathrm{Si}$ consideramos que ambas compañías estaban asociadas en el grupo River Plate Trust, resulta notoria la complementariedad de las trayectorias de rentabilidad de ambas. En el siguiente gráfico, las curvas de rentabilidad muestran el ciclo próspero de Mortgage Company desde su creación hasta 1930, mientras que el ciclo de auge de River Plate $\xi^{\circ}$ General Investment es menos acentuado durante la primera economía global y más prominente a partir de la segunda posguerra. Precisamente los elevados rendimientos del trust van a motivar sus planteos de independencia de la matriz RPTLA enunciados por Pilkington en los años 1940.

Con respecto a la cartera de inversiones de ambas compañías, se observa que hasta la Primera Guerra Mundial el portfolio estaba altamente concentrado en acciones que representaban activos en el Río de la Plata. En los años veinte, la cartera comenzó a diversificarse, sumando títulos de gobierno y luego, acciones de empresas industriales en Gran Bretaña. No obstante, en el segundo ciclo de auge de las firmas, el portfolio volvió a concentrarse: $87 \%$ de las inversiones correspondía a activos de firmas industriales británicas. ${ }^{40}$ Por lo tanto, en los ciclos expansivos, ambos portfolios se concentraron.

40. Es posible que ese porcentaje incluya activos de las filiales de multinacionales británicas fuera de Gran Bretaña, de modo que la distribución regional resulta engañosa, no así su concentración sectorial.

196
Esta observación nos resulta llamativa en tanto que en ese período primaba la idea de que la diversificación de los holdings permitía cambiar en forma flexible de un tipo de activos a otros para aprovechar más rápidamente las oportunidades emergentes. Esta estrategia confirmaría que los grupos británicos típicos invertían en un solo país y en una sola actividad como lo señalaron Jones y Wale en su estudio sobre las compañías británicas en Asia. ${ }^{41}$

La selección de valores bajo un patrón concentrado sectorial y geográficamente realizada por las compañías del grupo River Plate Trust muestra que las estrategias de los grupos de inversión británicos fueron equivalentes a las implementadas por los trusts de inversión de la segunda economía global un siglo más tarde. Este ejercicio de análisis confirma además que la inversión británica en Argentina y en Uruguay rendía dividendos mayores que si se hubieran invertido en el mercado interno, tal como lo plantearan Pollard y Edelstein para los países bajo dominio del imperio británico. ${ }^{42}$ Ningún trust cuya cartera de inversiones estuviera compuesta por valores asociados a activos en Gran Bretaña, obtuvo dividendos mayores a los generados por los trusts especializados en inversiones en el Río de la Plata entre 1900 y 1930. Esto se ve confirmado cuando comparamos los

41. JONES, Geoffrey, WALE, Judith, "Merchants as Business Groups: British Trading Companies in Asia before 1945", en The Business History Review, Cambridge, 1998, V. LXXII.

42. POLLARD, Sidney, "Capital Exports, 1870-1914: Harmful or Beneficial?", en Economic History Review, 1985, V. XXXVIII, No 4; EDELSTEIN, Michael, Overseas investment in the age of high imperialism: the United Kingdom, 1850 - 1914, Columbia University Press, New York, 1982. 
dividendos obtenidos por diferentes trusts de inversión durante ese período, pero también es corroborado por otros estudios de caso. Por ejemplo, Foreign \& Colonial Investment Trust cuyo portfolio mayoritariamente estaba formado por valores sobre activos en América del Norte, devengó una ganancia nominal promedio del $5.2 \%$ por acción entre $1880 \mathrm{y}$ 1913.

Los títulos de gobierno parecen haber sido más atractivos que las acciones a fines del siglo XIX, pero en el novecientos, las hipotecas primero y las acciones y los debentures corporativos daban un rendimiento superior a cualquier otra inversión. Los patrones del ciclo iniciado en la primera economía global perduraron aún después de su desintegración, al punto de que Sudamérica y particularmente Argentina, proveyó de recursos de capital al norte europeo durante la crisis de 1930. En consecuencia, confirmamos que el declive relativo de las inversiones en el Río de la Plata no se debió a la introducción de controles gubernamentales o de políticas proteccionistas activadas por la crisis del treinta, sino que la tendencia respondió más bien al agotamiento de un patrón de inversión orientado a obtener ganancias de la financiación del sector agropecuario y la industria urbana durante las fases de expansión económica en los países nuevos en condiciones de escasa competencia. La estrategia de replicar en Argentina y en Uruguay, la práctica de negocios antes desarrollada en Estados Unidos y en Nueva Zelanda, había resultado absolutamente exitosa y llegó a su fin sólo al cabo de sesenta años de vigencia cuando la Segunda Guerra Mundial abrió el paso a una nueva forma de acción estatal en las economías occidentales. 